\title{
An investigation of chromospheric activity spanning the Vaughan-Preston gap: impact on stellar ages $\star, \star \star$
}

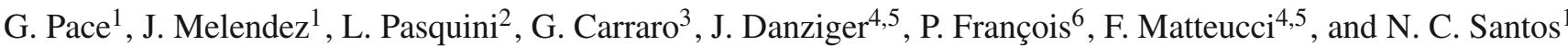 \\ ${ }^{1}$ Centro de Astrofisica, Universidade do Porto, Rua das Estrelas, 4150-762 Porto, Portugal \\ e-mail: gpace@astro.up.pt \\ 2 European Southern Observatory, Karl Schwarzschildstr. 2, Garching bei München, Germany \\ 3 European Southern Observatory, Casilla 19001, Santiago, Chile \\ 4 INAF, Osservatorio Astronomico di Trieste, via G.B. Tiepolo 11, 34131 Trieste, Italy \\ 5 Department of Astronomy, University of Trieste, via G.B. Tiepolo 11, 34131 Trieste, Italy \\ ${ }^{6}$ Observatoire de Paris, 64 Avenue de l'Observatoire, 75014 Paris, France
}

Received 17 March 2009 / Accepted 19 April 2009

\section{ABSTRACT}

\begin{abstract}
Context. Chromospheric activity is widely used as an age indicator for solar-type stars based on the early evidence that there is a smooth evolution from young and active to old and inactive stars. However, this transition may require modification as chromospheric activity is not a viable age indicator for stars older than $1 \mathrm{Gyr}$.

Aims. We analysed chromospheric activity in five solar-type stars in two open clusters, in order to study how chromospheric activity evolves with time.

Methods. We took UVES high-resolution, high S/N ratio spectra of 3 stars in IC 4756 and 2 in NGC 5822, which were combined with a previously studied data-set and reanalysed here. The emission core of the deep, photospheric Ca II K line was used as a probe of the chromospheric activity.

Results. All of the 5 stars in the new sample, including those in the 1.2 Gyr-old NGC 5822, have activity levels comparable to those of Hyades and Praesepe.

Conclusions. A likely interpretation of our data is that solar-type-star chromospheric activity, from the age of the Hyades until that of the Sun, does not evolve smoothly. Stars change from active to inactive, crossing the activity range corresponding to the Vaughan-Preston gap, on a time-scale that might be as short as 200 Myr. Evolution before and after such a transition is much less significant than cyclical and long-term variations. We show that data presented in the literature to support a correlation between age and activity could be also interpreted differently in the light of our results. Suggestions have been published that relevant stellar structures and/or dynamos are different for active and inactive stars. These provide a natural explanation for the observations presented here. More observations are required in order to strengthen our results, especially a long-term follow up of our two targets in the 1.2-Gyr old cluster NGC 5822.
\end{abstract}

Key words. stars: chromospheres

\section{Introduction}

It was already noticed in the early $60 \mathrm{~s}$ (Wilson 1963) that chromospheric activity decreases during the main sequence life time of late type stars like the Sun, and that the strength of the emission in the core of the broad and deep $\mathrm{Ca}$ II $\mathrm{H}$ and $\mathrm{K}$ lines is a reliable indicator of chromospheric activity level. From that time on, the possibility of using $\mathrm{H}$ and $\mathrm{K}$ emission to measure stellar ages has been widely explored and an impressive amount of data on stellar chromospheric activity has been collected, especially in the Mount Wilson program, started by Wilson (1968), and, more recently, during planet-search surveys (see e.g. Isaacson 2009). Unfortunately, chromospheric activity as an age indicator suffers a major limitation. Activity cycles equivalent to the 11-year long solar one, longer-term activity variations, which caused the Maunder-minimum in the Sun, and, in some stars, a

\footnotetext{
* Based on observations collected at the European Organisation for Astronomical Research in the Southern Hemisphere, Chile, during the observing run 073.D-0655.

$\star \star$ Table 1 is only available in electronic form

at http://www. aanda.org
}

hidden dependence on stellar mass (Cardini \& Cassatella 2007) and rotational modulation, strongly affect the level of activity. We believe that there is an even more severe limitation on the use of chromospheric activity as an age indicator: its decay almost stops after about $1.5 \mathrm{Gyr}$, therefore activity is not a reliable indicator of age for stars older than that.

Two papers support this view: Pace \& Pasquini (2004), hereafter Paper I, of which this study can be considered a continuation, and Lyra \& Porto de Mello (2005). Nevertheless, many other works pointed out the existence of a correlation between chromospheric activity and age, and many attempts were made to calibrate chromospheric ages (Soderblom et al. 1991; Donahue 1993; Lachaume et al. 1999). It is of primary importance to understand the limitations of chromospheric activity as a reliable age indicator, since it has been used to study, for example, the age-metallicity relation in the Galactic disk (Rocha-Pinto et al. 2000), and the age of stars hosting planetary systems (Saffe et al. 2005).

In order to understand how chromospheric activity decays, observations in solar-type stars in open clusters are the most appropriate tool, since their ages can be determined much more 
precisely than for field stars. The paucity of old open clusters in the solar neighbourhood hindered our progress until the advent of the 8-and 10-meter class telescopes, which made possible the acquisition of spectra of solar-type stars in several old and distant clusters at a resolution and a signal-to-noise ratio high enough to distinguish and resolve the emission core in the CaII K line. This is necessary to accurately correct for the absorption by interstellar lines of $\mathrm{Ca}$ II and thus to reliably measure chromospheric activity.

To the sample analysed in Paper I, we have added data on 5 solar-type stars in the open clusters NGC 5822 and IC 4756. We selected the two new target clusters mainly on the basis of their age, specifically because we wanted their ages to lie between those of the Hyades and Praesepe and those of IC 4651 and NGC 3680, studied by Salaris et al. (2004). As a result we have chromospheric activity data for 25 stars younger than 1 Gyr, 2 stars at about 1 Gyr, 7 stars at about $1.5 \mathrm{Gyr}$, and 6 at solar age.

The availability of UVES spectra allowed us to also accurately measure temperatures and metallicities for our target stars, which have been used in the present work, but the details of which will be published elsewhere.

\section{Observations and sample}

Target stars were selected to be high-probability single members of IC 4756 and NGC 5822 and to be as similar to the Sun as possible. The choice was made on the basis of the photometry by Herzog et al. (1975) for IC 4756 and by Twarog et al. (1993) for NGC 5822. The former study was made with photographic plates, and the cluster suffers from variable extinction, therefore the photometric precision was not good enough to exclude binaries with a high level of confidence. On the other hand, probabilities of membership published therein are based on a proper motion study which uses a time baseline of 43 years, therefore are quite accurate. Spectra were taken with the UVES spectrograph at the focus of the Kuyen telescope. Observations were carried out between April and September 2004. The wavelength coverage is from 3200 to $4600 \AA$ for the blue arm, and from 4800 to $6800 \AA$ for the red arm. The slit width for the former was set to $0.8 \mathrm{arcsec}$, and for the latter to $0.4 \mathrm{arcsec}$, giving a resolution of $R \approx 60000$ and $R \approx 100000$ respectively (Dekker et al. 2000). Data were reduced using the UVES pipeline (Modigliani et al. 2004).

From 2 to 7 spectra were obtained for each star. Radial velocity measurements were used to strengthen single-member selection. Details will be given elsewhere (Pace et al. 2009, in preparation). The final new sample consists of 3 stars in IC 4756 and 2 in NGC 5822.

To the best of our knowledge, Salaris et al. (2004) provide the most recent set of age evaluations for a sample of open clusters that includes all of those analysed by us. They find an age of 0.7 Gyr for both Hyades and Praesepe, 0.8 Gyr for IC 4756, 1.2 Gyr for NGC 5822, 1.4 Gyr for NGC 3680, 1.7 Gyr for IC 4651, and 4.3 Gyr for M 67.

\section{Data analysis and results}

As an indicator of chromospheric activity we used $F_{\mathrm{K}}^{\prime}$, i.e. the energy flux of the $\mathrm{Ca}$ II $\mathrm{K}$ line emitted per unit surface in the chromosphere. It is common practice in the literature to normalise chromospheric fluxes to bolometric emission and to use the logarithmic scale (Noyes et al. 1984). Hence we also used as a proxy of the chromospheric activity: $\log R_{\mathrm{K}}^{\prime}=\log \left(F_{\mathrm{K}}^{\prime} / \sigma T_{\mathrm{eff}}^{4}\right)$.

Normalisation of the spectra was done as in Paper I. From Paper I we also took 1- $\AA$ K index measurements for NGC 3680 , IC 4651 and M 67. Index measurements in Praesepe and Hyades stars were slightly revised with respect to the values published in Paper I: the new indices are about $15 \mathrm{~m} \AA$ higher. The stars in IC 4756 and NGC 5822 showed the redshifted interstellar K absorption line which affected the chromospheric K-line feature. To measure the $1-\AA \mathrm{K}$ index in these stars, we integrated the normalised flux only in the uncontaminated part of their profile. The Praesepe stars are unaffected by IS absorption, thus allowing us to calculate a ratio between the flux measured over the $1-\AA$ and that measured over the portion of the feature which is unaffected by interstellar lines in all stars. The initial measures for the affected stars were then multiplied by this factor to give a final corrected $1-\AA \mathrm{K}$ index. The errors involved in the measurement of the $1-\AA \mathrm{K}$ index were evaluated to be within $6 \%$. The $1-\AA \mathrm{K}$ index and chromospheric activity measurements, along with stellar parameters, are given in Table 1.

In Fig. 1 we show the average cluster spectra for IC 4756 and NGC 5822 and, for comparison, that of Praesepe and the solar one. The interstellar feature is apparent in IC 4756 and NGC 5822.

For the Sun, we used the $1-\AA \mathrm{K}$ index measurements made by White \& Livingston (1981). They monitored solar chromospheric activity from the first minimum to the maximum of the 21 st solar-activity cycle.

Subtraction of the photospheric contribution to the $1-\AA \mathrm{K}$ index was made as follows. For the Sun, we adopted the same photospheric correction as in Paper I, computed using a solarphotosphere synthetic spectrum (courtesy of P. Bonifacio). For the other stars, the photospheric contribution was computed by scaling the solar photospheric contribution by a factor that depends on the stellar parameters. These scaling factors were computed using the spectral synthesis code of MOOG (Sneden 1973, version 2002), and Kurucz's grid of models (Kurucz 1993). Stellar parameters, namely temperature, gravity, microturbulence and metallicity, were known from Paulson et al. (2003) for the Hyades stars, and from Pace et al. (2008) for the remainder of the old sample, and from our chemical analysis for the new sample (Pace et al. 2009, in preparation).

In order to transform the $1-\AA \mathrm{K}$ index, which is an equivalent width, into an intrinsic flux, namely $F_{\mathrm{K}}^{\prime}$, we proceeded as in Paper I, except that we did not use published photometry, but we transformed stellar temperatures and metallicities into $B-V$ colours inverting Eq. (1) in Sousa et al. (2008). For the Sun we adopted $B-V=0.65$ mag (Pasquini et al. 2008). The use of stellar temperatures instead of published colours avoids the cluster-to-cluster bias introduced by the error in the cluster reddening.

Because of the way we measured the index in the new sample of stars, we preferred not to use a triangular filter in the integration of the index, as done, for instance, in Paulson et al. (2002). Owing also to the lack of common standards, we could not make our $\log R_{\mathrm{K}}^{\prime}$ coincident with the widely used chromospheric activity indicator $\log R_{\mathrm{HK}}^{\prime}$.

\section{Discussion}

In Fig. 2 we plot the temperature of the stars versus their chromospheric activity. This is done using as an indicator of chromospheric activity both $\log R_{\mathrm{K}}^{\prime}$ (left panel) and $F_{\mathrm{K}}^{\prime}$ (right panel). 


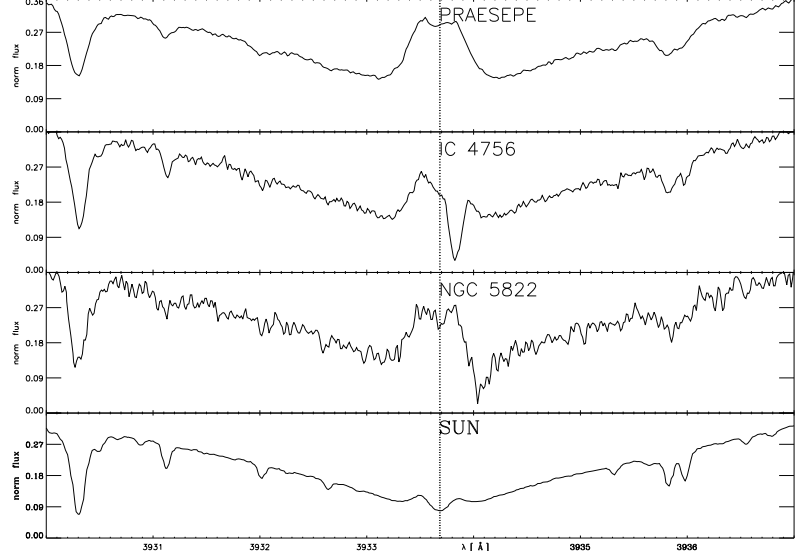

Fig. 1. The mean cluster Ca II K-line emission of IC 4756 and NGC 5822 is compared with that of Praesepe and with the Sun. All the spectra are normalised in the same way. Note the narrow IS absorption lines in the spectra of IC 4756 and NGC 5822.

The data are those shown in Table 1. The distribution of chromospheric activities is markedly bimodal: the more active stars are those in the Hyades, Praesepe, IC 4756, and NGC 5822, while the inactive stars are those in IC 4651, NGC 3680, and M 67 and the Sun. Within either group there is little difference between stars belonging to different clusters, although any small correlation with age using averages within the active group would be obscured by the cyclic range in individual stars. But the two groups are separated by a wide range of activities in which only two stars lie. This range coincides with what is known as the Vaughan-Preston gap, an underpopulated chromosphericactivity range identified by Vaughan \& Preston (1980) using a large sample of stars in the solar neighbourhood.

According to these ages and to the data shown in Fig. 2, no strong age-activity correlation appears to exist after $0.7 \mathrm{Gyr}$, just a rapid transition from active to inactive that occurs in a very short time, namely $200 \mathrm{Myr}$, between the age of NGC 5822 and that of NGC 3680. With this data it seems unlikely that a strong age-activity relationship could be masked by the scantiness of the data, or by the activity variations. Thus we can confidently conclude that chromospheric activity drops much faster between the ages of NGC 5822 and NGC 3680 than it does in the half Gyr before and in the 4 Gyr after. We would expect most of the stars in the gap to be young stars in an inactive phase or old stars in an active phase rather than stars at a certain age. In our sample we have one in either category. Two alternative explanations might be considered. Either our two targets in NGC 5822 were both in an exceptionally active phase when we observed them, or the age difference between the oldest active and the youngest inactive cluster is much larger than that claimed by Salaris et al. (2004). In the former case, NGC 5822 solar-type members would normally lie in the middle of the Vaughan-Preston gap. Owing to the extremely small sample of stars at this crucial age, it is well worth follow-up spectroscopic observations of the two NGC 5822 members to accurately measure their time-averaged chromospheric activity, and to add to the sample single members in the interesting temperature range. The possibility that the age of NGC 5822 has been over-estimated by Salaris et al. (2004) might be seriously considered because it was not determined directly, but by means of a photospheric index calibrated for a sample of 11 clusters. But in this case its age would need to have been overestimated by almost a factor of two.
According to Pace et al. (2008), Paulson et al. (2003) and the chemical analysis of the present sample of stars, the metallicity of the clusters studied ranges from $[\mathrm{Fe} / \mathrm{H}]=-0.04$ dex in NGC 3680 to $[\mathrm{Fe} / \mathrm{H}]=0.27 \mathrm{dex}$ in Praesepe. Since the $3 \mathrm{co}-$ eval active clusters have similar levels of chromospheric activity despite having different metallicities $([\mathrm{Fe} / \mathrm{H}]=0.01,0.13$ and $0.27 \mathrm{dex}$ ), metallicity does not appear to play a major role within the limited range of values spanned by our clusters.

Chromospheric activity undergoes large variations on short time-scales. For instance, the chromospheric activity of the Sun, from the highest activity peak to the Maunder minimum, spans a range that goes from the lower boundary of the Vaughan-Preston gap to slightly above the level of the most inactive stars (only $8 \%$ of the solar neighbourhood stars less active than the lowest solar value). This range, according to the current calibration of the age-versus- $\log R_{H K}^{\prime}$ relationship, corresponds to an age interval from 2.5 to 8 Gyr (Henry et al. 1996). However, members of binary or multiple systems have a similar level of chromospheric activity. It is mainly this circumstance that led Soderblom et al. (1991) to claim that chromospheric activity correlates well with age and allows a calibration. In the light of the results presented here, we suggest alternative explanations should be sought.

Another calibration of the chromospheric activity evolution with time is that of Lachaume et al. (1999). From Fig. 4 therein, it can be seen that their result agrees with ours as far as inactive stars are concerned, i.e. chromospheric activity does not evolve after it has crossed the Vaughan-Preston gap, at least until the solar age. As far as active stars older than the Hyades are concerned, there are 4 to 6 data-points (for two stars it is not possible to say whether or not they are younger than the Hyades and therefore out of the range considered in the present study). For this group, the Pearson coefficient indicates a level of anticorrelation between age and $\log R_{H K}^{\prime}$ that is insignificant $(-0.27)$ or fair $(-0.57)$, depending on how many stars we consider. We conclude that these data are compatible with the conclusion that, within the age range from 0.7 to $1.2 \mathrm{Gyr}$ and from 1.4 Gyr to solar age, any age-activity relationship must be weaker than the short term variations.

From Mamajek \& Hillenbrand (2008) it is clear that the one open cluster that supports a strict monotonicity of the chromospheric activity time evolution (once short-time scale variations are smoothed out) in the age range between that of the Hyades and that of M 67 and the Sun, is NGC 752. This cluster, according to Salaris et al. (2004), is older than NGC 5822 and younger than NGC 3680, i.e. exactly in the range in which we expect the transition to occur. Therefore, again, these data are not incompatible with the scenario we suggest, in which the intermediate chromospheric activity level of NGC 752 would be due to its age.

The two groups of stars on opposite sides of the VaughanPreston gap differ not only in their chromospheric activity level, but also in its trend with the colour/temperature. This can be seen in Fig. 2, and it is much more evident when a larger spectral-type range is considered (see e.g. Mamajek \& Hillenbrand 2008). Furthermore, temporal variations of chromospheric activity are large and irregular for active stars and small and regular for inactive ones. Several attempts have been made to provide a physical explanation for this, for example Durney et al. (1981) proposed a transition from a complex to a simpler magnetic-field morphology which occurs at the time when the rotation decreases enough to reach a threshold value. More recently, Barnes (2003) detected two sequences in the period-versus-colour diagram of open clusters, and he associated them with two different rotation morphologies, intertwined with stellar magnetic fields. 

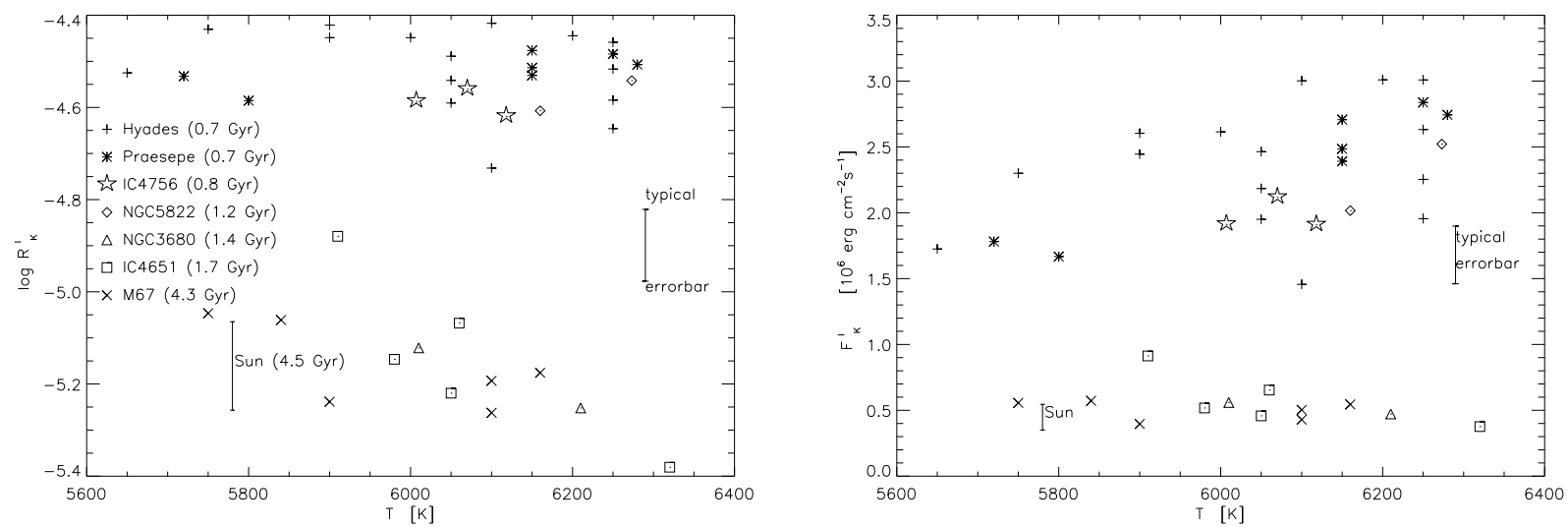

Fig. 2. Spectroscopic temperature versus chromospheric activity, using as a proxy of chromospheric activity both $\log R_{\mathrm{K}}^{\prime}\left(l e f t\right.$ panel) and $F_{\mathrm{K}}^{\prime}($ right panel). For the Sun, the bar corresponding to its full variation during a cycle is plotted. The typical error bar shows the typical uncertainty $( \pm 1 \sigma)$ evaluated from the error analysis, assuming an uncertainty in the temperature of $110 \mathrm{~K}$. Had we just added the counts to measure the 1 - $\AA \mathrm{K}$ index in the new sample stars, which gives a secure lower limit (see Sect. 3), we would have measured in the 2 NGC 5822 stars a value of log $R_{\mathrm{K}}^{\prime} \operatorname{lower}$ by only 0.09 and 0.07 . The ages come from Salaris et al. (2004).

Böhm-Vitense (2007) suggested a change of dynamo mechanism to explain the fact that stars occupy two very distinct sequences in a rotation period-versus-cycle period diagram. The kind of scenario in which a transition of the nature of the dynamo takes place at a specific point of the stellar lifetime could be used to explain the phenomena reported and discussed by us.

Acknowledgements. It is a pleasure to thank Boris Dintrans for long and useful discussions on the dynamo effect. We also thank the referee for useful comments. Data collected at ESO, VLT. This publication made use of data products from the WEBDA database, created by J.-C- Mermilliod and now operated at the institute for Astronomy of the University of Vienna. The SIMBAD astronomical database and the NASA's Astrophysics Data System Abstract Service have also been extensively used. G. P., J. M. and N. C. S. acknowledge the support of projects PTDC/CTE-AST/65971/2006 and PTDC-CTE/AST-66181/2006 of the Portuguese Fundação para a Ciência e a Tecnologia.

\section{References}

Barnes, S. A. 2003, ApJ, 586, 464

Böhm-Vitense, E. 2007, ApJ, 657, 486

Cardini, D., \& Cassatella, A. 2007, ApJ, 666, 393

Dekker, H., D’Odorico, S., Kaufer, A., Delabre, B., \& Kotzlowski, H. 2000, Proc. SPIE, 4008, 534

Donahue, R. A. 1993, Ph.D. Thesis,

Durney, B. R., Mihalas, D., \& Robinson, R. D. 1981, PASP, 93, 537

Isaacson, H. T. 2009, American Astronomical Society Meeting Abstracts, 213, \#408.07
Henry, T. J., Soderblom, D. R., Donahue, R. A., \& Baliunas, S. L. 1996, AJ, 111, 439

Herzog, A. D., Sanders, W. L., \& Seggewiss, W. 1975, A\&AS, 19, 211

Lachaume, R., Dominik, C., Lanz, T., \& Habing, H. J. 1999, A\&A, 348, 897

Lyra, W., \& Porto de Mello, G. F. 2005, A\&A, 431, 329

Kurucz, R. L. 1993, ATLAS9 Stellar Atmosphere Programs (Kurucz CD-ROM No. 13)

Mamajek, E. E., \& Hillenbrand, L. A. 2008, ApJ, 687, 1264

Modigliani, A., Mulas, G., Porceddu, I., \& Dammiani, F. 2004, VLT-MAN-ESO19500-3016

Noyes, R. W., Hartmann, L. W., Baliunas, S. L., Duncan, D. K., \& Vaughan, A. H. 1984, ApJ, 279, 763

Pace, G., \& Pasquini, L. 2004, A\&A, 426, 1021

Pace, G., Pasquini, L., \& François, P. 2008, A\&A, 489, 403

Pasquini, L., Biazzo, K., Bonifacio, P., Randich, S., \& Bedin, L. R. 2008, A\&A, 489, 677

Paulson, D. B., Saar, S. H., Cochran, W. D., \& Hatzes, A. P. 2002, AJ, 124, 572

Paulson, D. B., Sneden, C., \& Cochran, W. D. 2003, AJ, 125, 3185

Rocha-Pinto, H. J., Maciel, W. J., Scalo, J., \& Flynn, C. 2000, A\&A, 358, 850

Saffe, C., Gómez, M., \& Chavero, C. 2005, A\&A, 443, 609

Salaris, M., Weiss, A., \& Percival, S. M. 2004, A\&A, 414, 163

Sneden, C. 1973, ApJ, 184, 839

Soderblom, D. R., Duncan, D. K., \& Johnson, D. R. H. 1991, ApJ, 375, 722

Sousa, S. G., Santos, N. C., Mayor, M., et al. 2008, A\&A, 487, 373

Twarog, B. A., Anthony-Twarog, B. J., \& McClure, R. D. 1993, PASP, 105, 78

Vaughan, A. H., \& Preston, G. W. 1980, PASP, 92, 385

White, O. R., \& Livingston, W. C. 1981, ApJ, 249, 798

Wilson, O. C. 1963 , ApJ, 138, 832

Wilson, O. C. 1968, ApJ, 153, 221 
Table 1. The sample with the 3 different chromospheric indices.

\begin{tabular}{|c|c|c|c|c|}
\hline Star & $\overline{T_{\text {eff }}}$ & $\begin{array}{l}1-\AA \mathrm{K} \\
\text { index } \\
{[\mathrm{m} \AA]}\end{array}$ & $\begin{array}{c}F_{\mathrm{K}}^{\prime} \\
{\left[10^{6} \mathrm{erg} /\right.} \\
\left.\mathrm{cm}^{2} \mathrm{~s}\right]\end{array}$ & $\begin{array}{r}\log R_{\mathrm{K}}^{\prime} \\
{[\mathrm{dex}]}\end{array}$ \\
\hline \multicolumn{5}{|c|}{ Hyades, 0.7 Gyr } \\
\hline van Bueren 1 & 6250 & 180 & $1.95 \pm 0.17$ & $-4.65 \pm 0.04$ \\
\hline van Bueren 2 & 6050 & 223 & $2.18 \pm 0.24$ & $-4.54 \pm 0.05$ \\
\hline van Bueren 10 & 6100 & 281 & $3.00 \pm 0.31$ & $-4.42 \pm 0.04$ \\
\hline van Bueren 15 & 5750 & 308 & $2.30 \pm 0.34$ & $-4.43 \pm 0.06$ \\
\hline van Bueren 17 & 5650 & 272 & $1.73 \pm 0.29$ & $-4.53 \pm 0.07$ \\
\hline van Bueren 18 & 5900 & 294 & $2.60 \pm 0.33$ & $-4.42 \pm 0.05$ \\
\hline van Bueren 31 & 6200 & 261 & $3.01 \pm 0.28$ & $-4.44 \pm 0.04$ \\
\hline van Bueren 49 & 6050 & 207 & $1.95 \pm 0.21$ & $-4.59 \pm 0.05$ \\
\hline van Bueren 52 & 6050 & 248 & $2.46 \pm 0.27$ & $-4.49 \pm 0.05$ \\
\hline van Bueren 65 & 6250 & 202 & $2.25 \pm 0.20$ & $-4.58 \pm 0.04$ \\
\hline van Bueren 66 & 6250 & 253 & $3.01 \pm 0.27$ & $-4.46 \pm 0.04$ \\
\hline van Bueren 73 & 6000 & 270 & $2.61 \pm 0.30$ & $-4.45 \pm 0.05$ \\
\hline van Bueren 88 & 6250 & 228 & $2.63 \pm 0.23$ & $-4.52 \pm 0.04$ \\
\hline van Bueren 97 & 5900 & 279 & $2.44 \pm 0.31$ & $-4.45 \pm 0.05$ \\
\hline van Bueren 118 & 6100 & 161 & $1.46 \pm 0.15$ & $-4.73 \pm 0.04$ \\
\hline \multicolumn{5}{|c|}{ Praesepe, 0.7 Gyr } \\
\hline KW 49 & 6150 & 233 & $2.39 \pm 0.25$ & $-4.53 \pm 0.04$ \\
\hline KW 100 & 6150 & 255 & $2.71 \pm 0.28$ & $-4.48 \pm 0.04$ \\
\hline KW 208 & 6280 & 238 & $2.74 \pm 0.25$ & $-4.51 \pm 0.04$ \\
\hline KW 326 & 5800 & 234 & $1.67 \pm 0.25$ & $-4.59 \pm 0.06$ \\
\hline KW 368 & 5720 & 270 & $1.78 \pm 0.29$ & $-4.53 \pm 0.07$ \\
\hline KW 392 & 6250 & 248 & $2.84 \pm 0.26$ & $-4.48 \pm 0.04$ \\
\hline KW 418 & 6150 & 239 & $2.48 \pm 0.26$ & $-4.51 \pm 0.04$ \\
\hline \multicolumn{5}{|c|}{ NGC 3680, 1.4 Gyr } \\
\hline Eggen 60 & 6010 & $95 \pm 5$ & $0.56 \pm 0.09$ & $-5.12 \pm 0.07$ \\
\hline Eggen 70 & 6210 & $84 \pm 10$ & $0.47 \pm 0.16$ & $-5.25 \pm 0.14$ \\
\hline \multicolumn{5}{|c|}{ IC $4651,1.7 \mathrm{Gyr}$} \\
\hline AMC 1109 & 6060 & $102 \pm 10$ & $0.65 \pm 0.14$ & $-5.07 \pm 0.10$ \\
\hline AMC 2207 & 6050 & $85 \pm 10$ & $0.46 \pm 0.13$ & $-5.22 \pm 0.13$ \\
\hline AMC 4220 & 5910 & $133 \pm 20$ & $0.91 \pm 0.24$ & $-4.88 \pm 0.12$ \\
\hline AMC 4226 & 5980 & $93 \pm 15$ & $0.52 \pm 0.18$ & $-5.15 \pm 0.15$ \\
\hline Eggen 45 & 6320 & $74 \pm 10$ & $0.38 \pm 0.16$ & $-5.38 \pm 0.18$ \\
\hline \multicolumn{5}{|c|}{ M $67,4.3 \mathrm{Gyr}$} \\
\hline Sanders 746 & 5750 & $108 \pm 20$ & $0.56 \pm 0.20$ & $-5.05 \pm 0.16$ \\
\hline Sanders 1048 & 5900 & $85 \pm 10$ & $0.40 \pm 0.12$ & $-5.24 \pm 0.13$ \\
\hline Sanders 1092 & 6160 & $88 \pm 15$ & $0.54 \pm 0.22$ & $-5.18 \pm 0.17$ \\
\hline Sanders 1255 & 5840 & $105 \pm 20$ & $0.57 \pm 0.22$ & $-5.06 \pm 0.17$ \\
\hline Sanders 1283 & 6100 & $82 \pm 15$ & $0.43 \pm 0.21$ & $-5.26 \pm 0.21$ \\
\hline Sanders 1287 & 6100 & $90 \pm 10$ & $0.50 \pm 0.14$ & $-5.19 \pm 0.12$ \\
\hline \multicolumn{5}{|c|}{ NGC 5822, 1.2 Gyr } \\
\hline TATM 11003 & 6160 & $197 \pm 8$ & $2.02 \pm 0.22$ & $-4.61 \pm 0.05$ \\
\hline TATM 11014 & 6270 & $217 \pm 7$ & $2.52 \pm 0.24$ & $-4.54 \pm 0.04$ \\
\hline \multicolumn{5}{|c|}{ IC $4756,0.8 \mathrm{Gyr}$} \\
\hline HER 165 & 6070 & $211 \pm 7$ & $2.12 \pm 0.23$ & $-4.56 \pm 0.05$ \\
\hline HER 240 & 6010 & $206 \pm 6$ & $1.91 \pm 0.22$ & $-4.59 \pm 0.05$ \\
\hline HER 97 & 6120 & $190 \pm 9$ & $1.91 \pm 0.22$ & $-4.62 \pm 0.05$ \\
\hline \multicolumn{5}{|c|}{ Solar cycle 21, from White \& Livingston (1981) } \\
\hline Sun & 5780 & $95 \pm 10$ & $0.45 \pm 0.10$ & \\
\hline
\end{tabular}

